Elsevier required licence: $(c)<2018>$. This manuscript version is made available under the CC-BY-NC-ND 4.0 license http://creativecommons.org/licenses/by-nc-nd/4.0/ 


\section{A plastic-damage model for concrete under compression}

by

Atila Sarikaya $^{1}$ and R. Emre Erkmen ${ }^{2}$

\footnotetext{
${ }^{1} \mathrm{PhD}$ Student, School of Civil and Environmental Engineering, University of Technology Sydney, Broadway, NSW 2007, Australia

${ }^{2}$ Senior Lecturer, School of Civil and Environmental Engineering, University of Technology Sydney, Broadway, NSW 2007, Australia
}

${ }^{*}$ Corresponding Author: Dr R.E. Erkmen, School of Civil and Environmental Engineering, University of Technology Sydney, Broadway, NSW 2007, Australia

Phone: +61 29514 9769; Email: emre.erkmen@uts.edu.au

Submitted to: International Journal of Mechanical Sciences, $19^{\text {th }}$ of July 2018

Revised Manuscript Submitted: $14^{\text {th }}$ of October 2018

Accepted: $20^{\text {th }}$ of October 2018 


\begin{abstract}
A phenomenological model for plain concrete under compression is formulated within the framework of the coupled elastoplastic-damage theory. Phenomenological elastoplastic-damage models have been widely used for concrete because of their capability of representing both the permanent inelastic deformations and the degradation of material moduli beyond the elastic range. The essential contribution introduced in this paper is the proposed partitioning of the strain tensor within the coupled elastoplasticdamage framework which simplifies the selection of the failure surface and the potential function. Proposed partitioning permits the use of single failure criterion and single potential surface that are effective for both damage and plasticity models during inelastic deformations. Therefore, the coupled elastoplastic-damage model can be easily calibrated to fit the observed concrete behaviour based on well-established non-associated plasticity rules for concrete. The proposed approach also simplifies the numerical procedure by eliminating iterations that is required to equilibrate the stresses in plastic and damage components of the model. The numerical implementation is explained, and the results predicted by the model are compared with experimental data provided in the literature.
\end{abstract}

KEYWORDS: $\quad$ Concrete; Inelastic model; Phenomenological model; Coupled damage-plasticity; Cyclic loading 


\section{Introduction}

Coupled elastoplastic-damage models have been applied extensively for the description of the progressive failure of materials such as concrete, geomaterials, woods, steel and composites. These models have the capability of representing both the permanent inelastic deformations due to plastic component and the degradation of elastic moduli due to damage component. While the framework for plasticity is well established with the additive decomposition of the strain tensor into elastic and plastic parts, flow rule and Kuhn-Tucker conditions, approach to damage has been rather diverse. One of the main distinction between the alternative formulations is the way the strain tensor is decomposed. An initial attempt for merging elastoplastic and damage constitutive models can be found in Lemaitre (1985), where the total strain was partitioned as the elastic and plastic strains, while the effect of damage on the elasticity modulus was considered based on energy dissipation and thermodynamic principles. Simo and Ju (1987) employed the effective stress and effective strain concepts for the damage component and developed strain and stress based formulations and algorithms for the coupled elastoplastic-damage constitutive modelling. They also applied their theories for the simulation of the concrete material behaviour. Later, Ju (1989) developed an energy-based coupled elastoplastic-damage modelling approach. Constitutive models that are capable of coupling elastoplasticity and damage were also used for plain concrete by Meschke et al. (1998), in which the inelastic strains were decomposed into plastic and damage components and their share was determined based on a scalar parameter which was calibrated based on experimental results. Algorithmic issues in their analysis have also been discussed in Meschke et al. (1998). Lee and Fenves (1998) successfully coupled plastic and damage models to simulate the cyclic behaviour of concrete in both tension and compression. Vaz and Owen (2001) developed an algorithm for failure predictions of multi-fracturing materials based on the elastoplastic-damage modelling approach. Al-Rub and Kim (2010) used a coupled plasticity-damage model for the simulation of the fracture process of plain concrete. Brünig (2003) developed an elastoplastic-damage model to capture the phenomenological behaviour of metals considering finite 
strains and anisotropic damage development. Later, Brünig and Michalski (2017) used the model to capture the behaviour of concrete in both tension and compression. Other coupled plasticity and damage models include the works of Benallal et al. (1988), Lubliner et al. (1989), Hansen and Schreyer (1994), Doghri (1995), Luccioni et al. (1996), Jason et al. (2006), Grassl and Jirasek (2006), Wu et al. (2006), Einav et al. (2007) and Voyiadjis et al. (2008).

In this work, we follow the idea which proposes the partitioning of the total strain into elastic, plastic and damage strain components. According to the authors' knowledge, the work of Klisinski and Mroz (1988) was the first in which the constitutive equations were constructed by partitioning the total strain into the elastic, plastic and damage strain components. Yazdani and Schreyer (1990), Armero and Oller (2000), Al Rub and Voyiadjis (2003), and Brünig (2003) also partitioned the total strain considering a damage strain component. Armero and Oller (2000), however, introduced a novel framework in which the sharing of the total strain was determined based on equilibrium conditions between the updated stresses of the plastic and damage components of the model. In their framework, the problem for damage is posed in a similar form to plasticity, in which given the damage strain, flow rule and Kuhn-Tucker conditions are applied to determine the damage evolution and stress update within the damage component. It is interesting to note that in Armero and Oller (2000), damage strain component is reversible in parallel to the elastic strain, however, the damage evolution is irreversible which is represented by a separate damage variable. This definition of reversible damage strain differs from irreversible damage strain used in alternative studies, e.g. Al Rub and Voyiadjis (2003), and Brünig (2003), which naturally leads to alternative expressions for constitutive equations. Ibrahimbegovic and his co-workers adopted the framework proposed by Armero and Oller (2000) for the analysis of concrete in Ibrahimbegovic et al. (2008), and mild steel in Ayhan et al. (2013). In this study, however, by defining an a-priori relationship between the total strain and the damage strain components based on the damage variable, we depart from Armero and Oller (2000) in order to provide a simpler and more efficient computational framework. We limit our approach to isotropic damage development. 
The outline of this paper is as follows: In the next section, we review the coupled elastoplastic-damage model and set forth the relationship between damage and plasticity components. In Section 3, the numerical implementation of the coupled model is presented in the discrete form. Section 4 is allocated to the specifics of the adopted concrete material model. At Section 5, we present validation examples of the numerical methods; and we conclude at Section 6.

\section{Coupled elastoplastic-damage phenomenological model}

\subsection{Basic Hypothesis}

Following Armero and Oller (2000) and Ibrahimbegovic (2009), the coupled damage and plasticity constitutive equations can be built on three basic hypotheses: additive decomposition of the total strain field, the strain energy and finally the plasticity and damage initiation criteria.

- The additive decomposition implies that the total deformation can be decomposed into elastic part $\boldsymbol{\varepsilon}_{e}$, plastic part $\boldsymbol{\varepsilon}_{p}$ and damage part $\boldsymbol{\varepsilon}_{d}$ as $\boldsymbol{\varepsilon}=\boldsymbol{\varepsilon}_{e}+\boldsymbol{\varepsilon}_{p}+\boldsymbol{\varepsilon}_{d}$.

- The strain energy stored during deformation can be written as the sum of elastic and damage strain energies plus the energies due to hardening effects of plastic and damage parts:

$$
\Psi\left(\boldsymbol{\varepsilon}, \boldsymbol{\varepsilon}_{p}, \kappa_{p}, \phi, \boldsymbol{\varepsilon}_{d}, \kappa_{d}\right)=\Psi^{e}\left(\boldsymbol{\varepsilon}_{e}\right)+\Psi^{d}\left(\boldsymbol{\varepsilon}_{d}, \phi\right)+\Xi^{p}\left(\kappa_{p}\right)+\Xi^{d}\left(\kappa_{d}\right)
$$

where hardening effects can be accounted with the hardening potentials for the plastic behaviour $\Xi^{p}\left(\kappa_{p}\right)$ and damage behaviour $\Xi^{d}\left(\kappa_{d}\right)$, which are functions of hardening variables $\kappa_{p}$ and $\kappa_{d}$ for plasticity and damage, respectively.

Elastic strain energy can be written as $\Psi^{e}\left(\boldsymbol{\varepsilon}_{e}\right)=\boldsymbol{\sigma}^{\mathrm{T}} \boldsymbol{\varepsilon}_{e}-\chi^{e}$ in which $\chi^{e}(\boldsymbol{\sigma})=\frac{1}{2} \boldsymbol{\sigma}^{\mathrm{T}} \mathbf{E}^{-1} \boldsymbol{\sigma}$, where $\boldsymbol{\sigma}$ is the stress vector and $\mathbf{E}$ is the elastic constitutive matrix. It should be noted that elastic response is assumed linear.

The damage strain energy is $\Psi^{d}\left(\boldsymbol{\varepsilon}_{d}, \phi\right)=\boldsymbol{\sigma}^{\mathrm{T}} \boldsymbol{\varepsilon}_{d}-\chi^{d}(\boldsymbol{\sigma}, \phi)$ in which $\chi^{d}(\boldsymbol{\sigma}, \phi)=\frac{1}{2} \boldsymbol{\sigma}^{\mathrm{T}} \phi \mathbf{E}^{-1} \boldsymbol{\sigma}$ is the complementary damage energy, where $\phi$ is the damage parameter indicating the level of isotropic damage state. The reason for introducing $\Psi^{d}$ is to accommodate the degradation in the 
material stiffness. The presence of $\Psi^{d}$ identifies the recoverable nature of the damage strains. The damage parameter $\phi$ can be written in terms of the reduction factor as $\phi=\varphi /(1-\varphi)$, where $\varphi \in[0,1]$ is a measure of reduction in the load carrying area.

- The elastic domain can be specified as the domain in stress space where no change of internal variables, i.e., $\boldsymbol{\varepsilon}_{p}, \kappa_{p}, \phi, \boldsymbol{\varepsilon}_{d}, \kappa_{d}$, takes place. The boundaries of the domain is determined by the plastic failure criterion and the damage failure criterion with

$$
\Phi^{p}\left(\boldsymbol{\sigma}, q_{p}\right) \leq 0 \text { and } \Phi^{d}\left(\boldsymbol{\sigma}, q_{d}\right) \leq 0 \text { where } q_{p}=-\frac{\partial \Xi^{p}}{\partial \kappa_{p}}, q_{d}=-\frac{\partial \Xi^{d}}{\partial \kappa_{d}}
$$

Within the framework of Armero and Oller (2000), the plastic and damage failure criteria are assumed independent from each other; however, herein we assume that plasticity and damage evolve simultaneously. Therefore, only one criterion is used, that is $\Phi^{p}\left(\boldsymbol{\sigma}, q_{p}\right)=\Phi^{d}\left(\boldsymbol{\sigma}, q_{d}\right)$.

\subsection{An interpretation of the model by Armero and Oller (2000)}

An interpretation of the coupled plastic-damage model proposed by Armero and Oller (2000) in the uniaxial case is given in Fig. 1.
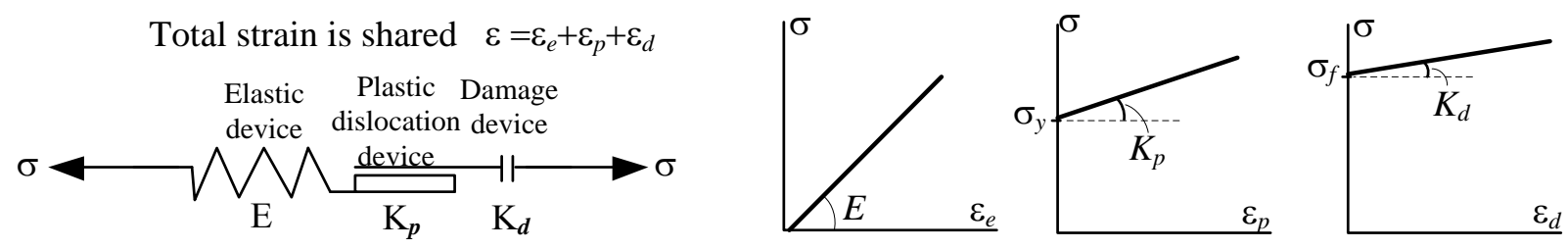

Fig. 1. Interpretation of the coupled model of Armero and Oller (2000), where plastic and damage devices are connected in series.

In Fig. 1, the elastic, plastic dislocation and damage devices are connected in series consistent with the kinematic decomposition of the strain, where $E, K_{p}, K_{d}, \sigma_{y}$ and $\sigma_{f}$ are the elastic modulus, plastic hardening modulus, damage hardening modulus, yield stress limit and the fracture stress limit, respectively. While the plastic device introduces irreversible strain $\varepsilon_{p}$ after unloading, the damage device introduces reversible strains $\varepsilon d$, due to reduced resistance of the device after damage. This physical interpretation allows the mechanisms for damage and plasticity to be considered independent of each 
other within the framework of Armero and Oller (2000). We diverge from the assumption of independent damage and plasticity evolution mechanisms by introducing a kinematic constraint in Section 2.6.

\subsection{Thermo-dynamic considerations}

Here, we would like to introduce the relationship between stress, strain and hardening components considering the local form of the second principle of thermodynamics. That is, the total inelastic dissipation is always non-negative, i.e., $\mathrm{d} \Omega=\boldsymbol{\sigma}^{\mathrm{T}} \mathrm{d} \boldsymbol{\varepsilon}-\mathrm{d} \Psi \geq 0$. Subsequently, one obtains (Ibrahimbegovic 2009)

$$
\left(\boldsymbol{\sigma}^{\mathrm{T}}-\frac{\partial \Psi^{e \mathrm{~T}}}{\partial \boldsymbol{\varepsilon}_{e}}\right) \mathrm{d} \boldsymbol{\varepsilon}_{e}+\boldsymbol{\sigma}^{\mathrm{T}} \mathrm{d} \boldsymbol{\varepsilon}_{p}-\frac{\partial \Xi^{p}}{\partial \kappa_{p}} \mathrm{~d} \kappa_{p}+\mathrm{d} \boldsymbol{\sigma}^{\mathrm{T}}\left(\frac{\partial \chi^{d}}{\partial \boldsymbol{\sigma}}-\boldsymbol{\varepsilon}_{d}\right)+\frac{\partial \chi^{d}}{\partial \phi} \mathrm{d} \phi-\frac{\partial \Xi^{d}}{\partial \kappa_{d}} \mathrm{~d} \kappa_{d} \geq 0
$$

where $\boldsymbol{\sigma}^{\mathrm{T}} \mathrm{d} \boldsymbol{\varepsilon}_{d}=\mathrm{d}\left(\boldsymbol{\sigma}^{\mathrm{T}} \boldsymbol{\varepsilon}_{d}\right)-\mathrm{d} \boldsymbol{\sigma}^{\mathrm{T}} \boldsymbol{\varepsilon}_{d}$ has been used. Considering hyperelastic behaviour for the elastic device, the stress can be obtained from the elastic strain energy, i.e., $\boldsymbol{\sigma}-\frac{\partial \Psi^{e}}{\partial \boldsymbol{\varepsilon}_{e}}=0$. Thus the first term vanishes in Eq. (1), in which $\mathrm{d} \Omega^{p}=\boldsymbol{\sigma}^{\mathrm{T}} \mathrm{d} \boldsymbol{\varepsilon}_{p}-\frac{\partial \Xi^{p}}{\partial \kappa_{p}} \mathrm{~d} \kappa_{p}$ and $\mathrm{d} \Omega^{d}=\frac{\partial \chi^{d}}{\partial \phi} \mathrm{d} \phi-\frac{\partial \Xi^{d}}{\partial \kappa_{d}} \mathrm{~d} \kappa_{d}$ are non-negative plastic and damage dissipations, respectively. On the other hand, the damage strain is defined through stress and the current value of damage compliance, i.e.,

$$
\boldsymbol{\varepsilon}_{d}=\frac{\partial \chi^{d}}{\partial \boldsymbol{\sigma}}
$$

By using Eq. (2) and substituting into Eq. (1), the dissipation inequality reduces to

$$
\boldsymbol{\sigma}^{\mathrm{T}} \mathrm{d} \boldsymbol{\varepsilon}_{p}+q_{p} \mathrm{~d} \kappa_{p}+\frac{\partial \chi^{d}}{\partial \phi} \mathrm{d} \phi+q_{d} \mathrm{~d} \kappa_{d} \geq 0
$$

where $q_{p}=-\frac{\partial \Xi^{p}}{\partial \kappa_{p}}$ and $q_{d}=-\frac{\partial \Xi^{d}}{\partial \kappa_{d}}$ have been defined. In associated plasticity, maximization of the expression $\boldsymbol{\sigma}^{\mathrm{T}} \mathrm{d} \boldsymbol{\varepsilon}_{p}+q_{p} \mathrm{~d} \kappa_{p}$ for plastic energy dissipation in Eq. (3) produces the flow rule. However, for pressure sensitive frictional materials such as concrete, non-associated flow rule produces more accurate results in plasticity based models. Therefore, in the following, we adopt a non-associated flow rule for plasticity. It can be shown that under non-associated flow rule, the energy dissipation stays non- 
negative, hence thermo-dynamically consistent, e.g. (Houlsby and Puzrin 2000). The damage energy dissipation can be written from Eq. (3) as $\frac{\partial \chi^{d}}{\partial \phi} \mathrm{d} \phi+q_{d} \mathrm{~d} \kappa_{d}$ and its non-negativeness is assumed herein.

\subsection{Plastic model}

By using the non-associated flow rule, the plastic strain increment can be written as

$$
\mathrm{d} \boldsymbol{\varepsilon}_{p}=\mathrm{d} \lambda_{p} \mathbf{b}
$$

where $\mathbf{b}=\frac{\partial \Theta^{p}\left(\boldsymbol{\sigma}, q_{p}\right)}{\partial \boldsymbol{\sigma}}$ is the direction of the plastic strain increment, $\Theta^{p}\left(\boldsymbol{\sigma}, q_{p}\right)$ is the potential function for plasticity and $\mathrm{d} \lambda_{p}$ is the proportionality factor. Note that a negative proportionality factor $\mathrm{d} \lambda_{p}$ would imply plastic unloading which cannot occur. There is only elastic unloading allowed in which case the proportionality factor $\mathrm{d} \lambda_{p}$ is zero. The flow rule indicates that during the loading which causes plastic deformations, the stress increments should be tangential to the potential surface. Since the stress increments are produced by the elastic strain increments only, i.e., $\mathrm{d} \boldsymbol{\sigma}=\mathbf{E d} \boldsymbol{\varepsilon}_{e}$, the plastic strain increments do not produce stresses and their directions are normal to the potential surface. On the other hand, from the consistency condition, when plastic flow occurs, the stresses remain on the plastic failure surface, i.e.,

$$
\mathrm{d} \Phi^{p}=\frac{\partial \Phi^{p^{\mathrm{T}}}}{\partial \boldsymbol{\sigma}} \mathrm{d} \boldsymbol{\sigma}+\frac{\partial \Phi^{p}}{\partial q_{p}} \mathrm{~d} q_{p}=0
$$

By using $\mathrm{d} \boldsymbol{\sigma}=\mathbf{E}\left(\mathrm{d} \boldsymbol{\varepsilon}-\mathrm{d} \boldsymbol{\varepsilon}_{p}-\mathrm{d} \boldsymbol{\varepsilon}_{d}\right)$ and $\mathrm{d} \boldsymbol{\varepsilon}_{p}=\mathrm{d} \lambda_{p} \mathbf{b}$ in the equation above, $\mathrm{d} \lambda_{p}$ becomes

$$
\mathrm{d} \lambda_{p}=\frac{\mathbf{a}^{\mathrm{T}} \mathbf{E}}{\mathbf{a}^{\mathrm{T}} \mathbf{E b}-\frac{\partial \Phi}{\partial \Phi_{p}} \frac{\partial q_{p} \partial \kappa_{p}}{\partial \kappa_{p} \partial \lambda_{p}}}\left(\mathrm{~d} \boldsymbol{\varepsilon}-\mathrm{d} \boldsymbol{\varepsilon}_{d}\right)
$$

in which $\kappa_{p}$ is the hardening parameter for plasticity and $\mathbf{a}=\frac{\partial \Phi^{p}}{\partial \boldsymbol{\sigma}}$ was used. From Eqs. (4) and (6), by substituting into the relation $\mathrm{d} \boldsymbol{\sigma}=\mathbf{E}\left(\mathrm{d} \boldsymbol{\varepsilon}-\mathrm{d} \boldsymbol{\varepsilon}_{p}-\mathrm{d} \boldsymbol{\varepsilon}_{d}\right)$ one obtains

$$
\mathrm{d} \boldsymbol{\sigma}=\left(\mathbf{E}-\frac{\mathbf{E b a}^{\mathrm{T}} \mathbf{E}}{\mathbf{a}^{\mathrm{T}} \mathbf{E b}-\frac{\partial \Phi^{p} \partial q_{p} \partial \kappa_{p}}{\partial q_{p} \partial \kappa_{p} \partial \lambda_{p}}}\right)\left(\mathrm{d} \boldsymbol{\varepsilon}-\mathrm{d} \boldsymbol{\varepsilon}_{d}\right)=\mathbf{C}^{e p}\left(\mathrm{~d} \boldsymbol{\varepsilon}-\mathrm{d} \boldsymbol{\varepsilon}_{d}\right)
$$




\subsection{Damage model}

The damage model can be cast in a similar form as the one given for plasticity (Armero and Oller 2000, Ibrahimbegovic et al. 2008), i.e.,

$$
\boldsymbol{\sigma} \mathrm{d} \phi=\mathrm{d} \lambda_{d} \mathbf{E b}
$$

which can be interpreted as the evolution equation of the damage model with $d \lambda_{d}$ as the proportionality factor. From the consistency condition, when damage occurs, the stresses remain on the damage failure surface, i.e.,

$$
\mathrm{d} \Phi^{d}=\frac{\partial \Phi^{d^{\mathrm{T}}}}{\partial \boldsymbol{\sigma}} \mathrm{d} \boldsymbol{\sigma}+\frac{\partial \Phi^{d}}{\partial q_{d}} \mathrm{~d} q_{d}=0
$$

Differentiation of Eq. (2), and using $\phi \mathrm{d} \boldsymbol{\sigma}=\mathrm{d}(\phi \boldsymbol{\sigma})-\boldsymbol{\sigma} \mathrm{d} \phi=\mathbf{E d}\left(\frac{\partial \chi^{d}}{\partial \boldsymbol{\sigma}}\right)-\boldsymbol{\sigma} \mathrm{d} \phi$, together with Eq. (8) produces

$$
\mathrm{d} \boldsymbol{\sigma}=\phi^{-1} \mathbf{E d} \boldsymbol{\varepsilon}_{d}-\phi^{-1} \mathbf{E} \mathbf{b} \mathrm{d} \lambda_{d}
$$

By using Eqs. (9) and (10) and the relation $\mathbf{a}=\frac{\partial \Phi^{d}}{\partial \boldsymbol{\sigma}}, \mathrm{d} \lambda_{d}$ can be obtained as

$$
\mathrm{d} \lambda_{d}=\frac{\mathbf{a}^{\mathrm{T}} \mathbf{E} \phi^{-1} \mathrm{~d} \varepsilon_{d}}{\mathbf{a}^{\mathrm{T}} \mathbf{E} \phi^{-1} \mathbf{b}-\frac{\partial \Phi^{d}}{\partial q_{d}} \frac{\partial q_{d}}{\partial \kappa_{d}} \frac{\partial \kappa_{d}}{\partial \lambda_{d}}}
$$

Substituting Eqs. (11) into Eq. (10) produces,

$$
\mathrm{d} \boldsymbol{\sigma}=\left(\mathbf{E} \phi^{-1}-\frac{\mathbf{E b a}^{\mathrm{T}} \mathbf{E} \phi^{-2}}{\mathbf{a}^{\mathrm{T}} \mathbf{E} \phi^{-1} \mathbf{b}-\frac{\partial \Phi^{d}}{\partial q_{d}} \frac{\partial q_{d}}{\partial \kappa_{d}} \frac{\partial \kappa_{d}}{\partial \lambda_{d}}}\right) \mathrm{d} \boldsymbol{\varepsilon}_{d}=\mathbf{K}^{e d} \mathrm{~d} \boldsymbol{\varepsilon}_{d}
$$

It should be noted that since we have assumed the same failure surface expressions for both damage and plasticity models, i.e., $\Phi^{p}\left(\boldsymbol{\sigma}, q_{p}\right)=\Phi^{d}\left(\boldsymbol{\sigma}, q_{d}\right)$, a in Eq. (11) is identical to that in Eq. (6). Similarly, due to the use of the same potential function in plasticity and damage models $\mathbf{b}$ in Eq. (8) is identical to that in Eq. (4).

\section{6. $\quad$ Plastic-damage coupling}

According to the plastic-damage model of Armero and Oller (2000), when both plasticity and damage models are active, the stress increments in plastic and damage models are equal. This is because as 
indicated in Fig. 1, the plastic and damage components are connected in series. Thus, by equating Eq. (7) to Eq. (12) and eliminating d $\boldsymbol{\varepsilon}_{d}$ one obtains

$$
\mathrm{d} \boldsymbol{\sigma}=\mathbf{K}^{e d}\left[\mathbf{K}^{e d}+\mathbf{C}^{e p}\right]^{-1} \mathbf{C}^{e p} \mathrm{~d} \boldsymbol{\varepsilon}
$$

Here, our motivation is to be able to use a single failure surface for both plasticity and damage models. In order to be able to use a single failure surface at any step of the analysis, the evolution of the internal parameters of plastic and damage models need to be linked in a certain way. For this purpose, we propose a relationship between the increments of the total strain tensor and the damage strain tensor as

$$
\mathrm{d} \boldsymbol{\varepsilon}_{d}=\frac{\phi}{(1+\phi)} \mathrm{d} \boldsymbol{\varepsilon}
$$

Considering that damage strain occurs due to reduced resistance after damage which results with the reduction in stress as $\mathrm{d} \boldsymbol{\sigma}=(1-\varphi) \mathbf{E} \mathrm{d} \boldsymbol{\varepsilon}$, the damage strain increment is then considered herein as the strain required to compensate the stress reduction due to damage, i.e., $\mathrm{d} \boldsymbol{\varepsilon}_{d}=\varphi \mathrm{d} \boldsymbol{\varepsilon}$. The proposed relationship between the increments of the total strain and the damage strain in Eq. (14), is consistent with this definition since $\varphi=\phi /(1+\phi)$. It should be noted that in literature alternative a-priori imposed relations between the total strain and the damage strain have been considered by Meschke et al. (1998). Using Eq. (14) in Eqs. (7) and (12) produces

$$
\mathbf{K}^{e d}=\phi^{-1} \mathbf{C}^{e p}
$$

It should be noted that in order to be able to refer to the same failure surface for both plastic and damage considerations, in addition to Eq. (14), the hardening parameters are needed to be linked as

$$
\frac{\partial q_{d}}{\partial \kappa_{d}}=\phi^{-1} \frac{\partial q_{p}}{\partial \kappa_{p}}
$$

so that Eqs. (7) and (12) can be directly equated. By using Eqs. (6), (11), (14) and (16), one obtains

$$
\mathrm{d} \lambda_{d}=\phi \mathrm{d} \lambda_{p}
$$

It should be noted in obtaining Eq. (17), it was assumed that $\mathrm{d} q_{d}=\mathrm{d} q_{p}$ and

$$
\mathrm{d} \kappa_{d}=\phi \mathrm{d} \kappa_{p} .
$$


The consistency of the total damage strain expression in Eq. (2) with the damage strain rate in Eq. (14) can be shown by noting that the kinematic condition in Eq. (14), is indeed equivalent to $\varepsilon_{\mathrm{d}}=\varphi \boldsymbol{\varepsilon}-\varphi \varepsilon_{\mathrm{p}}$, because the last three terms in the derivative, i.e., $d \boldsymbol{\varepsilon}_{\mathrm{d}}=\varphi \mathrm{d} \boldsymbol{\varepsilon}+\mathrm{d} \varphi \boldsymbol{\varepsilon}-\varphi \mathrm{d} \boldsymbol{\varepsilon}_{\mathrm{p}}-\mathrm{d} \varphi \boldsymbol{\varepsilon}_{\mathrm{p}}$ vanish. This can be shown by employing Eq. (2), the constitutive relation $\boldsymbol{\sigma}=\mathbf{E}\left(\boldsymbol{\varepsilon}-\boldsymbol{\varepsilon}_{\mathrm{p}}-\boldsymbol{\varepsilon}_{\mathrm{d}}\right)$, and Eqs. (8) and (17), i.e., $\boldsymbol{\sigma d} \phi=\phi \mathbf{E d} \boldsymbol{\varepsilon}_{\mathrm{p}}$. It is interesting to note that the kinematic condition, i.e., $\boldsymbol{\varepsilon}_{\mathrm{d}}=\varphi\left(\boldsymbol{\varepsilon}-\boldsymbol{\varepsilon}_{\mathrm{p}}\right)$ is in-line with the hypothesis of strain equivalence (e.g., Lemaitre 1985) as the stress-strain relationship of damaged material, i.e., $\boldsymbol{\sigma}=\mathbf{E}\left(\boldsymbol{\varepsilon}-\boldsymbol{\varepsilon}_{\mathrm{p}}-\boldsymbol{\varepsilon}_{\mathrm{d}}\right)$ can be exchanged with the stress-strain relationship of the fictitious undamaged state, i.e., $\boldsymbol{\sigma}=(1-\varphi) \mathbf{E}\left(\boldsymbol{\varepsilon}-\boldsymbol{\varepsilon}_{\mathrm{p}}\right)$ in which plastic strain component needs to be considered as in Lee and Fenves (1998). Based on the kinematic condition employed in Eq. (14) or equivalently $\boldsymbol{\varepsilon}_{d}=\varphi\left(\boldsymbol{\varepsilon}-\boldsymbol{\varepsilon}_{p}\right)$, once the functions $\Phi^{p}\left(\boldsymbol{\sigma}, q_{p}\right)$ and $\Phi^{d}\left(\boldsymbol{\sigma}, q_{d}\right)$ are selected as the same expression they change with the same amount for a stress increment of $\mathrm{d} \boldsymbol{\sigma}$. This allows us to use single failure criterion for both damage and plasticity models during inelastic deformations at any stage.

\section{Computational algorithm}

For numerical computations, procedures based on finite increments are needed, where the problem is to compute the internal variables which will provide an admissible stress field for a given strain increment $\Delta \varepsilon_{n}$ of step $n$. In the following, the closest-point projection algorithm is adopted for the numerical calculations.

\subsection{Plastic computations}

As the first step, the residual stress vector at local iteration $i$ can be calculated as

$$
\mathbf{r}^{p i}=\boldsymbol{\sigma}^{p i}-\left(\boldsymbol{\sigma}_{n}+\mathbf{E} \Delta \boldsymbol{\varepsilon}_{n}-\Delta \lambda_{p}{ }^{i} \mathbf{E b}^{i}\right)
$$

where $\boldsymbol{\sigma}_{n}$ is the last converged stress at the end of previous global step, and $\boldsymbol{\sigma}^{p i}$ is initially $\boldsymbol{\sigma}_{n}+\mathbf{E} \Delta \boldsymbol{\varepsilon}_{n}$, which is updated at each local iteration $i$. It should be noted that $\Delta \lambda_{p}{ }^{i}$ is initially zero, as the trial step is based on no plastic deformation assumption. The residual stress in Eq. (19) is then used to calculate the increment in the proportionality factor as 


$$
\delta \lambda_{p}^{i}=\frac{\Phi^{p i}-\mathbf{a}^{T i} \mathbf{R}^{p i} \mathbf{E}^{-1} \mathbf{r}^{p i}}{\mathbf{a}^{\mathrm{Ti}} \mathbf{R}^{p i} \mathbf{b}^{i}+K_{p}}
$$

where $K_{p}=\frac{\partial \Phi^{p}}{\partial q_{p}} \frac{\partial q_{p}}{\partial \kappa_{p}} \frac{\partial \kappa_{p}}{\partial \lambda_{p}}$ has been used. In Eq. (20), $\mathbf{R}^{p i}$ is defined as

$$
\mathbf{R}^{p i}=\left(\mathbf{E}^{-1} \mathbf{Q}^{p i}\right)^{-1}
$$

in which

$$
\mathbf{Q}^{p i}=\mathbf{I}+\Delta \lambda_{p}{ }^{i} \mathbf{E} \mathbf{H}_{\mathbf{b}}{ }^{i},
$$

and

$$
\mathbf{H}_{\mathbf{b}}{ }^{i}=\frac{\partial \mathbf{b}^{i}}{\partial \boldsymbol{\sigma}}
$$

In Eq. (20), $\Phi^{p i}$ is evaluated by using the last updated stress $\boldsymbol{\sigma}^{p i}$. The proportionality factor of plastic deformations can be then updated using

$$
\Delta \lambda_{p}^{i+1}=\Delta \lambda_{p}^{i}+\delta \lambda_{p}^{i}
$$

The increment in the stress vector can be calculated as

$$
\delta \boldsymbol{\sigma}^{p i}=-\mathbf{R}^{p i}\left(\mathbf{E}^{-1} \mathbf{r}^{p i}+\delta \lambda_{p}{ }^{i} \mathbf{b}^{i}\right)
$$

From Eq. (25), the stress vector can be updated as

$$
\boldsymbol{\sigma}^{p i+1}=\boldsymbol{\sigma}^{p i}+\delta \boldsymbol{\sigma}^{p i}
$$

and the plastic hardening parameter is updated as

$$
\delta \kappa_{p}=\delta \lambda_{p}{ }^{i} \boldsymbol{\delta}^{\mathrm{T}} \mathbf{b}^{i}
$$

It should be noted that the volumetric strain increment $\delta \kappa_{p}$ is adopted herein as the parameter of the hardening law following Grassl et al. (2002). In Eq. (27), $\boldsymbol{\delta}^{\mathrm{T}}$ is the Kronecker delta operator, i.e., $\boldsymbol{\delta}^{\mathrm{T}} \mathbf{b}=b_{11}+b_{22}+b_{33}$.

\subsection{Damage computations}

Now, we will show that the same stress, i.e., $\boldsymbol{\sigma}^{d i+1}=\boldsymbol{\sigma}^{p i+1}$, can be generated following a damage-only procedure. Firstly, the residual stress vector at local iteration $i$ can be calculated as

$$
\mathbf{r}^{d i}=\boldsymbol{\sigma}^{d i}-\left(\boldsymbol{\sigma}_{n}+\mathbf{E} \Delta \boldsymbol{\varepsilon}_{n}-\Delta \lambda_{d}^{i} \phi^{-1} \mathbf{E} \mathbf{b}^{i}\right)
$$


where $\boldsymbol{\sigma}^{d i}$ is initially $\boldsymbol{\sigma}_{n}+\mathbf{E} \Delta \boldsymbol{\varepsilon}_{n}$. It can be verified that if $\Delta \lambda_{d}{ }^{i}=\phi \Delta \lambda_{p}{ }^{i}$, then residual stress vectors in Eqs. (19) and (28) are equal. In order to show that $\Delta \lambda_{p}{ }^{i+1}=\phi \Delta \lambda_{p}{ }^{i+1}$, it is sufficient to update the proportionality factor for damage by using the equation $\Delta \lambda_{d}{ }^{i+1}=\Delta \lambda_{d}{ }^{i}+\delta \lambda_{d}{ }^{i}$ in which $\delta \lambda_{d}{ }^{i}$ can be written as

$$
\delta \lambda_{d}^{i}=\frac{\Phi^{d i}-\mathbf{a}^{\mathrm{T} i} \mathbf{R}^{d i} \mathbf{E}^{-1} \mathbf{r}^{d i}}{\phi^{-1} \mathbf{a}^{\mathrm{T} i} \mathbf{R}^{d i} \mathbf{b}^{i}+K_{d}^{i}}
$$

where $K_{d}=\frac{\partial \Phi^{d}}{\partial q_{d}} \frac{\partial q_{d}}{\partial \kappa_{d}} \frac{\partial \kappa_{d}}{\partial \lambda_{d}}$ has been used. Under the assumptions given in Eqs. (16)-(18), it can be verified that $K_{d}=\phi^{-1} K_{p}$. In Eq. (29), matrix $\mathbf{R}^{d i}=\left(\mathbf{E}^{-1} \mathbf{Q}^{d i}\right)^{-1}$ is the same as in Eq. (21), because $\mathbf{Q}^{d i}$ is defined as

$$
\mathbf{Q}^{d i}=\mathbf{I}+\Delta \lambda_{d}^{i} \phi^{-1} \mathbf{E H}_{\mathbf{b}}{ }^{i}
$$

It should be noted that initially, $\Phi^{d i}=\Phi^{p i}$ since $\boldsymbol{\sigma}^{d i}=\boldsymbol{\sigma}^{p i}$, for the trial step as well as for later iterations. Therefore, from Eqs. (20) and (29), it can be verified that $\delta \lambda_{d}{ }^{i}=\phi \delta \lambda_{p}{ }^{i}$. On the other hand, the increment in the stress vector can be calculated as

$$
\delta \boldsymbol{\sigma}^{d i}=-\mathbf{R}^{d i}\left(\mathbf{E}^{-1} \mathbf{r}^{d i}-\delta \lambda_{d}^{i} \phi^{-1} \mathbf{b}^{i}\right)
$$

which is the same as in Eq. (25). Thus, same stress, i.e., $\boldsymbol{\sigma}^{d i+1}=\boldsymbol{\sigma}^{p i+1}$, is generated at the end of both stress update procedures. Therefore, in our procedure, there is no need for iterations to equate the stresses between the damage and plasticity models. In contrast, in Armero and Oller (2000) and Ibrahimbegovic et al. (2008), the partitioning of the total strain is determined as a result of the equilibrium between plastic and damage models. Since the plastic and damage devices are connected in series, as illustrated in Fig. 1, the updated stresses based on plastic and damage models should match, which generally requires iterations. 


\section{Specifics adopted for the concrete model}

The plastic model that determines the envelope curve for the stress-strain relationship consists of a potential surface, hardening law, which describes the deformation capacity in multiaxial compression, and a yield surface. Both the plastic potential and the yield surface are constituted by using the unified co-ordinates in the Haigh-Westergaard stress space, which are based on the stress invariants. The three co-ordinates $\xi, \rho$ and $\theta$ are given in terms of the stress components in Appendix A for convenience.

\subsection{Yield surface}

We employ the yield surface proposed by Menetrey and Willam (1995), i.e.,

$$
\Phi^{p}\left(\xi, \rho, \theta, \kappa_{p}\right)=(\sqrt{1.5} \rho)^{2}+q_{h}\left(\kappa_{p}\right) m\left(\frac{\rho}{\sqrt{6}} r(\theta)+\frac{\xi}{\sqrt{3}}\right)-q_{h}\left(\kappa_{p}\right) q_{s}\left(\kappa_{p}\right) \leq 0
$$

where $q_{h}$ and $q_{s}$ controls the shape and location of the loading surface and $m$ can be written as

$$
m=3 \frac{f_{c}^{2}-f_{t}^{2}}{f_{c} f_{t}} \frac{e}{e+1}
$$

in which $f_{c}$ is the uniaxial compressive strength, $f_{t}$ is the uniaxial tensile strength taken herein as $0.09 f_{c}$. The eccentricity defined by Menetrey and Willam (1995) can be written as

$$
e=\frac{1+\epsilon}{2-\epsilon}
$$

which is a formulation adopted by Jirasek and Bazant (2002), in which

$$
\epsilon=\frac{f_{t}}{f_{b}} \frac{f_{b}^{2}-f_{c}^{2}}{f_{c}^{2}-f_{t}^{2}}
$$

where $f_{b}$ is the equibiaxial compressive strength taken herein as $1.5 f_{c}^{-0.925}$. In Eq. (32), $r(\theta)$ is the polar radius, i.e.,

$$
r(\theta)=\frac{v(\theta)}{s(\theta)+t(\theta)}
$$

in which

$$
\begin{aligned}
& v(\theta)=4\left(1-e^{2}\right) \cos ^{2} \theta+(2 e-1)^{2} \\
& s(\theta)=2\left(1-e^{2}\right) \cos \theta \\
& t(\theta)=(2 e-1)\left[4\left(1-e^{2}\right) \cos ^{2} \theta+5 e^{2}-4 e\right]^{1 / 2}
\end{aligned}
$$




\subsection{Hardening and softening law}

Hardening and softening of concrete can be simulated by varying the shape and location of the loading surface during plastic flow. The variation is controlled by the hardening/softening parameter $\kappa_{p}$. During the hardening range, $q_{h}$ in Eq. (32) for concrete can be selected as (Papanikolaou and Kappos 2007)

$$
q_{h}\left(\kappa_{p}\right)=k_{o}+\left(1-k_{o}\right) \sqrt{1-\left(\frac{\varepsilon_{v o}^{p}-\kappa_{p}}{\varepsilon_{v o}^{p}}\right)^{2}}
$$

where

$$
k_{o}=\sigma_{c o} / f_{c}
$$

in which $\sigma_{c o}$ is the uniaxial concrete stress at the onset of plastic flow. In Eq. (40), $\varepsilon_{v o}^{p}$ is the threshold value for the volumetric plastic strain at uniaxial concrete strength, i.e.,

$$
\varepsilon_{v o}^{p}=\frac{f_{c}}{E_{c}}(1-2 v)
$$

where $E_{c}$ and $v$ are the Young's modulus and Poisson ratio for concrete, respectively. During softening range, $q_{s}$ in Eq. (32) for concrete can be selected as (Papanikolaou and Kappos 2007)

$$
q_{s}\left(\kappa_{p}\right)=\left(\frac{1}{1+\left(\frac{n_{1}-1}{n_{2}-1}\right)^{2}}\right)^{2}
$$

where $n_{1}=\frac{\kappa_{p}}{\varepsilon_{v o}^{p}}, n_{2}=\frac{\varepsilon_{v o}^{p}+t}{\varepsilon_{v o}^{p}}$ and $t=\frac{f_{c}}{15000}$. Note that $f_{c}$ is considered in MPa.

\subsection{Potential function}

The potential function is again written in Haigh-Westergaard stress space and adopted herein from Grassl et al. (2002), i.e.,

$$
\Theta^{p}\left(\xi, \rho, q_{p}\right)=-A\left(\frac{\rho}{\sqrt{q_{h}\left(\kappa_{p}\right) q_{s}\left(\kappa_{p}\right)}}\right)^{2}-B \frac{\rho}{\sqrt{q_{h}\left(\kappa_{p}\right) q_{s}\left(\kappa_{p}\right)}}+\frac{\xi}{\sqrt{q_{h}\left(\kappa_{p}\right) q_{s}\left(\kappa_{p}\right)}}
$$

in which

$$
A=\frac{\psi_{2}-\psi_{1}}{2\left(\rho_{1}-\rho_{2}\right)}
$$


and

$$
B=\rho_{1} \frac{\psi_{1}-\psi_{2}}{\left(\rho_{1}-\rho_{2}\right)}-\psi_{1}
$$

In Eqs. (45) and (46), $\rho_{1}$ and $\rho_{2}$ are the normalized deviatoric stress indicators at uniaxial and triaxial compressive strength, respectively, i.e.,

$$
\rho_{1}=\sqrt{\frac{2}{3}}
$$

and

$$
\rho_{2}=\sqrt{\frac{2}{3}} \frac{\left|f_{c c}-\sigma_{p c}\right|}{f_{c}}
$$

where $f_{c c}$ is the triaxial compressive strength taken herein as $4.333 f_{c}$ and $\sigma_{p c}$ is the lateral stress taken herein as $f_{c}$. On the other hand, $\psi_{1}$ and $\psi_{2}$ are the inclinations of the plastic strain vector under uniaxial and triaxial compressive strength, respectively, i.e.,

$$
\psi_{1}=\sqrt{2} \frac{\left|\varepsilon_{3 p u}-\varepsilon_{1 p u}\right|}{\varepsilon_{v o}^{p}}
$$

and

$$
\psi_{2}=\sqrt{2} \frac{\left|\varepsilon_{3 p c}-\varepsilon_{1 p c}\right|}{\varepsilon_{v o}^{p}}
$$

In Eq. (49), $\varepsilon_{3 p u}$ is the axial plastic strain component at uniaxial compressive strength, which can be calculated as

$$
\varepsilon_{3 p u}=\varepsilon_{c}-\frac{f_{c}}{E_{c}}
$$

and $\varepsilon_{1 p u}$ is the lateral plastic strain component at uniaxial compressive strength, which can be calculated as

$$
\varepsilon_{1 p u}=\varepsilon_{2 p u}=\frac{\varepsilon_{v o}^{p}-\varepsilon_{3 p u}}{2}
$$

In Eq. (51), $\varepsilon_{c}$ is the total strain in the axial direction at uniaxial compressive strength. In Eq. (50), $\varepsilon_{3 p c}$ is the axial plastic strain component at triaxial compressive strength, which can be calculated as

$$
\varepsilon_{3 p c}=\varepsilon_{c c}-\frac{1}{E_{c}}\left(f_{c c}-2 v \sigma_{p c}\right)
$$


and $\varepsilon_{1 p c}$ is the lateral plastic strain component at triaxial compressive strength, which can be calculated as

$$
\varepsilon_{1 p c}=\varepsilon_{2 p c}=\frac{\varepsilon_{v o}^{p}-\varepsilon_{3 p c}}{2}
$$

For concrete, it can be assumed that $\varepsilon_{c c}=\varepsilon_{c}\left(1+17 \frac{\sigma_{p c}}{f_{c}}\right)$, e.g. Papanikolaou and Kappos 2007 and generally $\sigma_{p c}$ is taken as $\sigma_{p c}=f_{c}$, and thus in Eq. (53) $\varepsilon_{c c}$ becomes $\varepsilon_{c c}=18 \varepsilon_{c}$.

\subsection{Evolution of the damage parameter}

The damage parameter $\phi$ is updated after every converged step. For this purpose, we have adopted the relationship given in Grassl and Jirasek (2006), i.e.,

$$
\varphi=\left(1-e^{-C \frac{\kappa_{p}}{\varepsilon_{v o}}}\right)
$$

In Eq. (55), $C$ is a parameter which is to be calibrated based on cyclic tests as shown in the next section.

It should be noted that under the assumption that $\frac{\varphi \boldsymbol{\sigma}^{\mathrm{T}}}{\boldsymbol{\sigma}^{\mathrm{T}} \mathbf{E}^{-\mathbf{1}} \boldsymbol{\sigma}} \mathrm{d} \boldsymbol{\varepsilon}_{p}=\frac{C}{\varepsilon_{v o}^{p}} \mathrm{~d} \kappa_{p}$, and considering Eq. (17), i.e., $\mathrm{d} \lambda_{d}=\phi \mathrm{d} \lambda_{p}$, Eq. (55) can be obtained as a solution of Eq. (8).

\section{Examples}

In order to illustrate the predictive capability of the proposed model, in this section, several numerical examples are analysed and compared with available experimental results from the literature. It should be noted that, in the selected cases the effects of anisotropic damage are not investigated, and thus the experiments are based on either monotonic loading or load cycles that are kept in the same direction. The developed numerical model is calibrated by adjusting the uniaxial compressive strength $f_{c}$, corresponding total strain at the uniaxial compressive strength $\varepsilon_{c}$, Young's modulus $E_{c}$, Poisson ratio $v$, the stress at the onset of plastic flow $k_{o}$ and the damage parameter $C$ in Eq. (55). In the following figures $\sigma_{3}$ is the axial stress, $\sigma_{1}$ and $\sigma_{2}$ are the lateral stresses, $\varepsilon_{3}$ is the axial strain, $\varepsilon_{1}$ and $\varepsilon_{2}$ are the lateral strains and $\varepsilon_{v}$ is the volumetric total strain. 
5.1. Comparisons with the experimental results of Karsan and Jirsa (1969)

In this example, the proposed model is compared with the experimental results of Karsan and Jirsa (1969). Given the experimental results, the model parameters are selected as shown in Table 1. As shown in Fig. 2, the envelope axial stress-strain curve is in perfect agreement with the experimental results. Fig. 3 shows the cyclic behaviour based on the test results of Karsan and Jirsa (1969) and that of our model. The reduction in the stiffness during the load cycles are very accurately captured with the model developed herein. It can be verified that the measured changes in the Young's modulus are in perfect agreement with the developed numerical solution. Comparison of the damage density evolution during the same cyclic test is presented in Fig. 4, in which the damage density $\varrho$ is calculated such that $\varrho=1-$ $\sqrt{1-\varphi}$

Table 1. Adjusted model parameters for the Karsan and Jirsa (1969) monotonic loading experiment.

\begin{tabular}{|rcccccc|}
\hline Experiment & $\boldsymbol{f}_{\boldsymbol{c}}(\mathbf{M P a})$ & $\boldsymbol{E}_{\boldsymbol{c}}(\mathbf{M P a})$ & $\boldsymbol{v}$ & $\boldsymbol{\varepsilon}_{\boldsymbol{c}}$ & $\boldsymbol{k}_{\boldsymbol{o}}$ & $\boldsymbol{C}$ \\
Monotonic Uniaxial & 27.4 & 31000 & 0.20 & -0.001996 & 0.14 & 0.355 \\
Cyclic Uniaxial & 28.0 & 31200 & 0.20 & -0.001880 & 0.14 & 0.355 \\
\hline
\end{tabular}

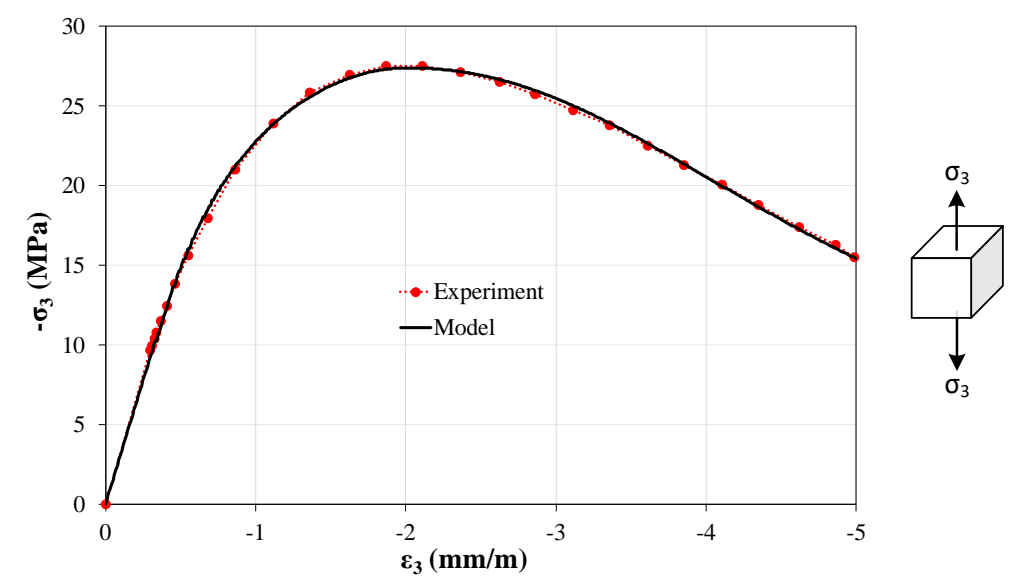

Fig. 2. Comparison with the stress-strain results of Karsan and Jirsa (1969) under monotonic uniaxial compressive loading. 


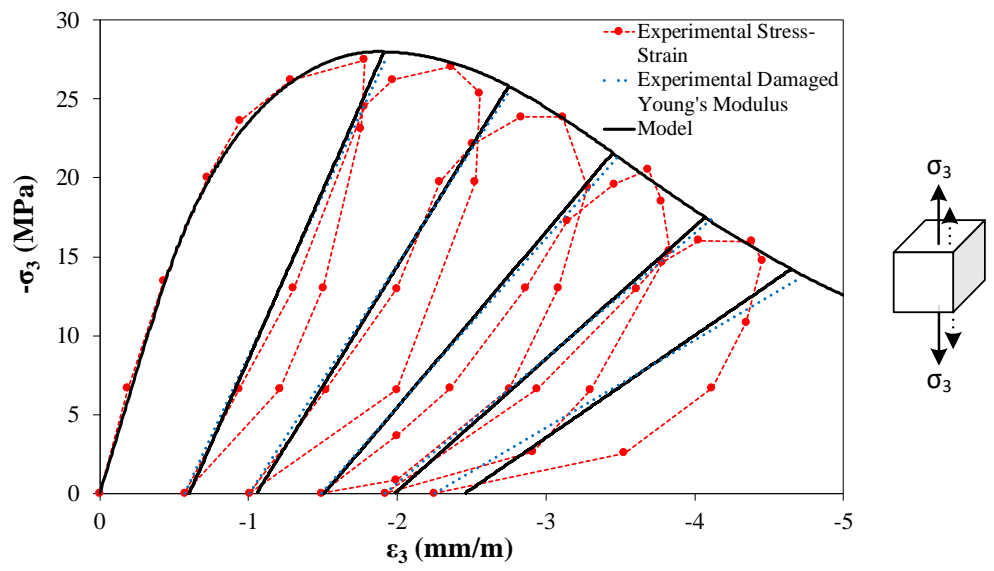

Fig. 3. Comparison with the stress-strain results of Karsan and Jirsa (1969) under cyclic uniaxial compressive loading.

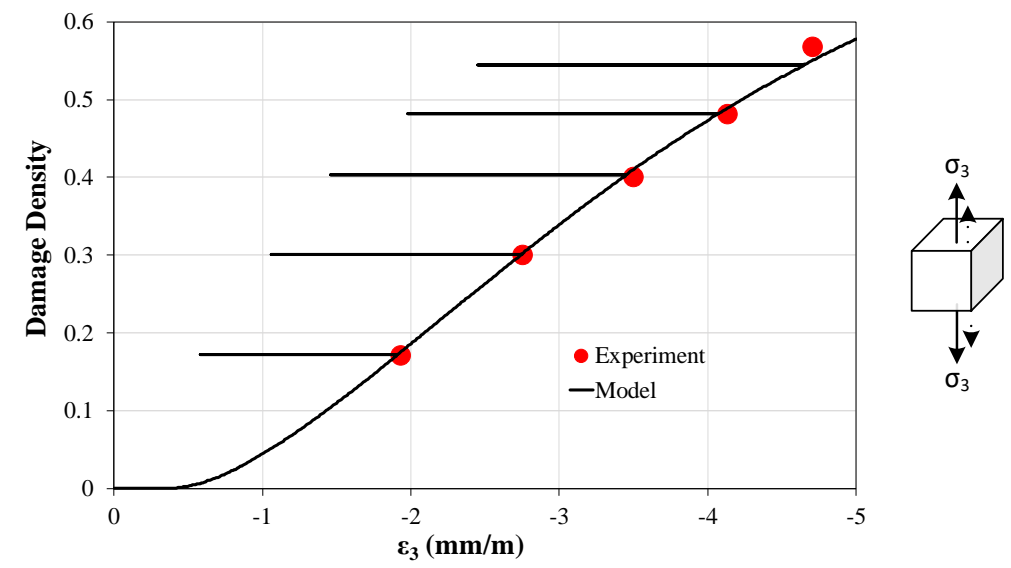

Fig. 4. Comparison with the damage density results of Karsan and Jirsa (1969) under cyclic uniaxial compressive loading.

\subsection{Comparisons with the experimental results of Dahl (1992)}

In this example, the proposed model is compared with the experimental results of Dahl (1992). The purpose is to show that the uniaxial stress-strain behaviour agrees well with the experimental results for different concrete grades. Fig. 5 shows that the proposed model can predict the uniaxial stress-strain relationships successfully both for normal and high strength concrete grades. The calibrated parameters for these experiments are given in Table 2. Note that the damage parameter $C$ has no influence on the stress-strain curve under monotonic loading, and therefore no specific value is shown in Table 2. 
Table 2. Adjusted model parameters for Dahl (1992) uniaxial loading experiments.

\begin{tabular}{|lccccc|}
\hline Experiment & $\boldsymbol{f}_{\boldsymbol{c}} \mathbf{( M P a )}$ & $\boldsymbol{E}_{\boldsymbol{c}} \mathbf{( M P a )}$ & $\boldsymbol{v}$ & $\boldsymbol{\varepsilon}_{\boldsymbol{c}}$ & $\boldsymbol{k}_{\boldsymbol{o}}$ \\
Strength: $21.7 \mathrm{MPa}$ & 21.7 & 19800 & 0.20 & -0.00310 & 0.05 \\
Strength: $31.7 \mathrm{MPa}$ & 31.7 & 26850 & 0.20 & -0.00251 & 0.05 \\
Strength: $50.3 \mathrm{MPa}$ & 50.3 & 29000 & 0.20 & -0.00259 & 0.05 \\
Strength: $65.0 \mathrm{MPa}$ & 65.0 & 32600 & 0.20 & -0.00253 & 0.05 \\
Strength: $93.9 \mathrm{MPa}$ & 93.9 & 40400 & 0.20 & -0.00267 & 0.08 \\
Strength: $105.7 \mathrm{MPa}$ & 105.7 & 42800 & 0.20 & -0.00274 & 0.08 \\
\hline
\end{tabular}

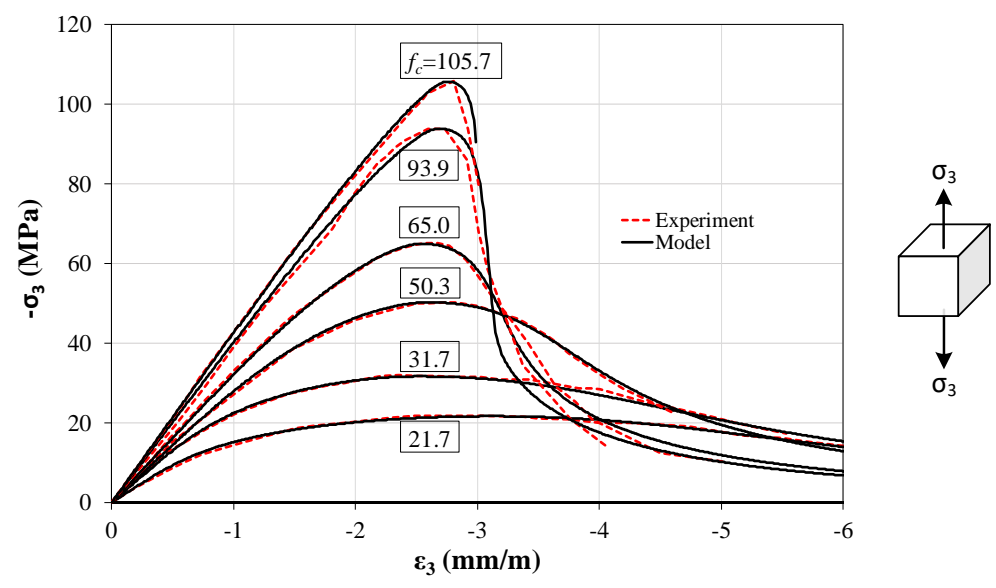

Fig. 5. Comparison with the stress-strain results of Dahl (1992) for normal and high-strength concrete under uniaxial loading.

\subsection{Comparisons with the experimental results of Kupfer et al. (1969)}

In order to illustrate that the proposed model can capture the concrete behaviour under multiaxial loading, the results of the model are compared with the experimental results of Kupfer et al. (1969). In Fig. 6 the axial stress versus axial strain, lateral strain and volumetric strain results are shown. The calibrated parameters for these experiments are given in Table 3. Similar to previous cases, the proposed model can capture the essential behaviour of the experimental results of Kupfer et al. (1969) under uniaxial loading. Also, as shown in Fig. 7 the model captures the experimental behaviour under equibiaxial loading. The axial stress versus axial strain and lateral strain relations are very close to the experimental curve. 
Table 3. Adjusted model parameters for Kupfer et al. (1969) uniaxial and equibiaxial loading experiments.

\begin{tabular}{|rccccc|}
\hline Experiment & $\boldsymbol{f}_{\boldsymbol{c}} \mathbf{( M P a )}$ & $\boldsymbol{E}_{\boldsymbol{c}} \mathbf{( M P a )}$ & $\boldsymbol{v}$ & $\boldsymbol{\varepsilon}_{\boldsymbol{c}}$ & $\boldsymbol{k}_{\boldsymbol{o}}$ \\
Uniaxial loading & 32.1 & 31500 & 0.20 & -0.00201 & 0.05 \\
Equibiaxial loading & 32.1 & 31500 & 0.20 & -0.00201 & 0.05 \\
\hline
\end{tabular}

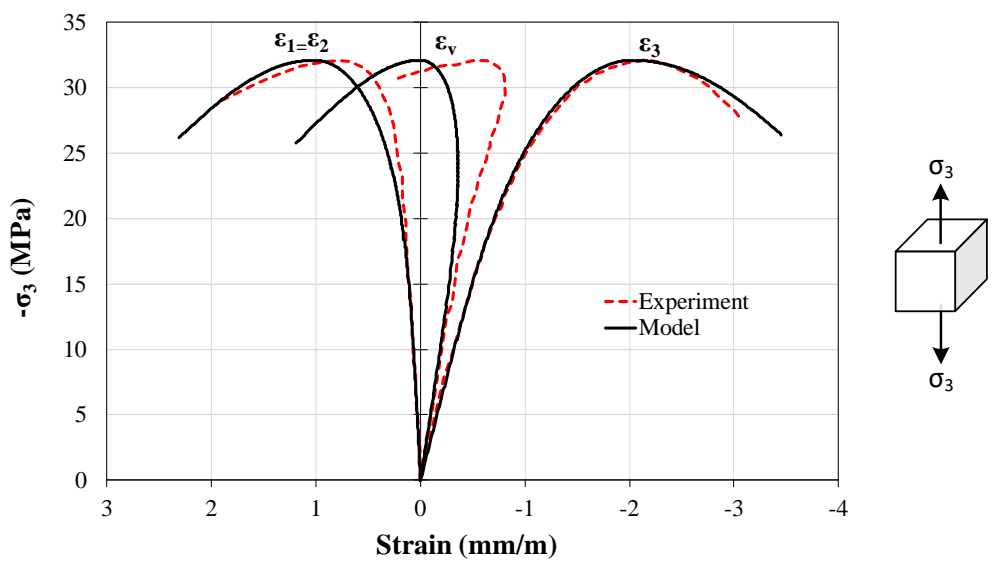

Fig. 6. Comparison with the experimental stress-strain results of Kupfer et al. (1969) for axial, lateral and volumetric strains under uniaxial compression.

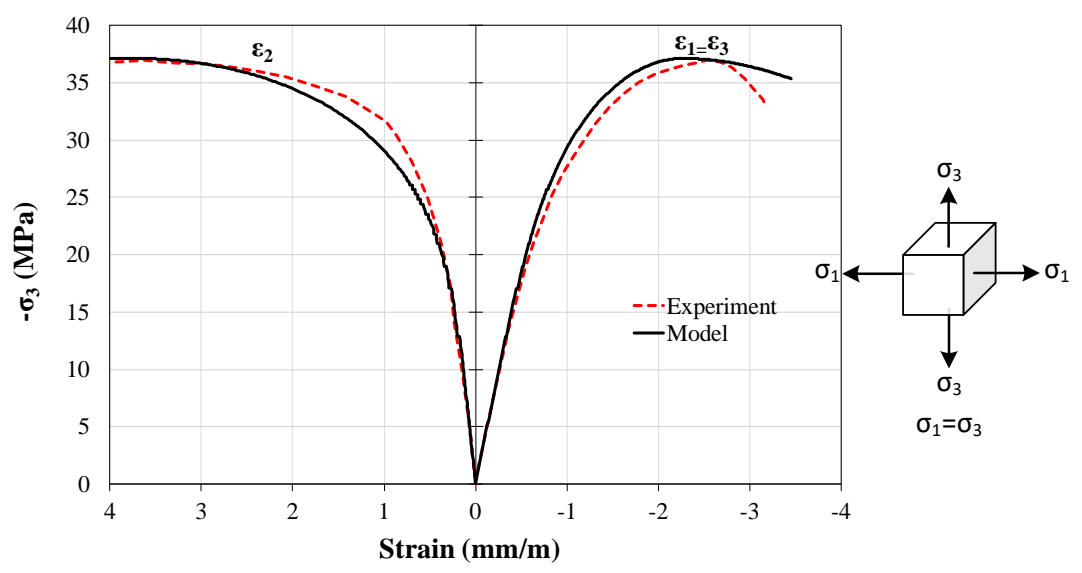

Fig. 7. Comparison with the experimental stress-strain results of Kupfer et al. (1969) in both axial and lateral directions under equibiaxial compression.

\section{4. $\quad$ Comparisons with the experimental results of Imran (1994)}

In order to illustrate that the proposed model can capture the concrete behaviour under multiaxial cyclic loading, the results are compared with the experimental results of Imran (1994). The calibrated parameters for these experiments are given in Table 4. Fig. 8 shows the triaxial monotonic loading stress- 
strain test results for various confinement pressure levels, and it can be verified that the proposed coupled damage plasticity model agrees well with those of the tests. In Fig. 9, axial and lateral stress-strain curves of a concrete specimen under cyclic loading are presented. The model damage parameter, in this case, is selected as $C=0.37$, and the degradation in the material modulus is captured.

Table 4. Adjusted model parameters for Imran (1994) triaxial loading experiments.

\begin{tabular}{|rcccccc|}
\hline Experiment & $\boldsymbol{f}_{\boldsymbol{c}}(\mathbf{M P a})$ & $\boldsymbol{E}_{\boldsymbol{c}} \mathbf{( M P a )}$ & $\boldsymbol{v}$ & $\boldsymbol{\varepsilon}_{\boldsymbol{c}}$ & $\boldsymbol{k}_{\boldsymbol{o}}$ & $\boldsymbol{C}$ \\
Monotonic triaxial loading & 47.13 & 29570 & 0.22 & -0.00282 & 0.05 & 0.37 \\
Cyclic triaxial loading & 45.00 & 29570 & 0.22 & -0.00282 & 0.05 & 0.37 \\
\hline
\end{tabular}

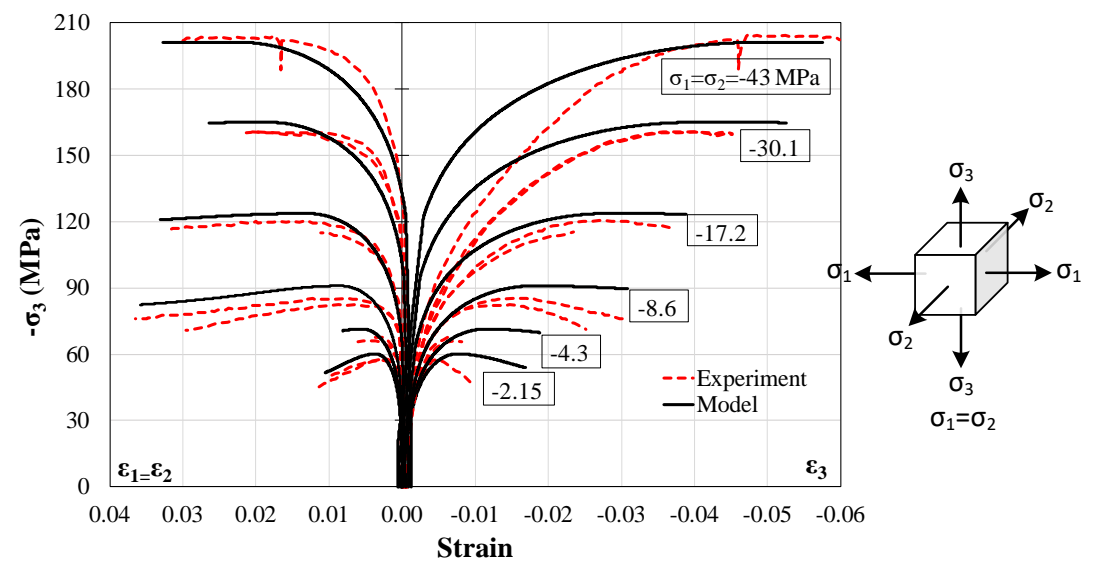

Fig. 8. Comparison with the experimental stress-strain results of Imran (1994) for normal concrete $(\mathrm{w} / \mathrm{c}=0.55)$ under triaxial compression and various confinement pressure levels.

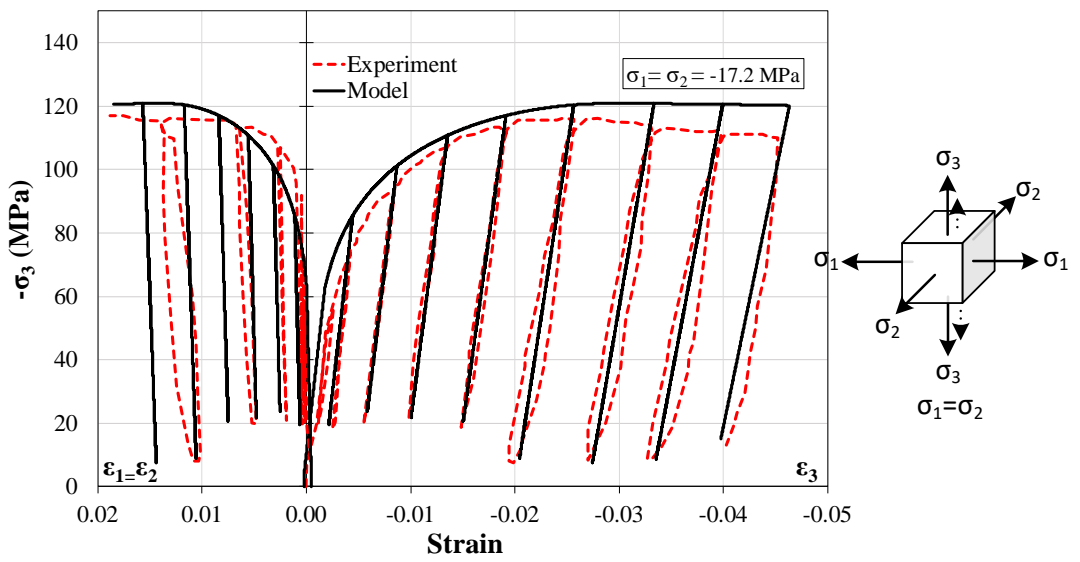

Fig. 9. Comparison with the experimental stress-strain results of Imran (1994) for normal concrete $(\mathrm{w} / \mathrm{c}=0.55)$ under cyclic axial loading with constant confinement pressure. 


\section{Conclusions}

For the numerical analyses of plain concrete under compression an efficient procedure that couples plasticity with isotropic damage is developed. The model captures permanent deformations due to plasticity model as well as degradation in the elastic response due to damage component. The developed approach allows a single failure surface and hardening/softening criterion to be adopted in order to characterise the inelastic behaviour of concrete. Selected examples include cyclic behaviour under uniaxial and confined loading as well as cases with different concrete strength. The results agree very well with those provided in the literature, in terms of the stress-strain relationship. The developed model is computationally efficient as it circumvents iterations which are required to equate the stresses between the coupled damage and plasticity models.

\section{APPENDIX A}

\section{Haigh-Westergaard co-ordinates}

The three co-ordinates $\xi, \rho$ and $\theta$ can be written as

$$
\begin{aligned}
& \xi=\frac{I_{1}}{\sqrt{3} f_{c}} \\
& \rho=\frac{\sqrt{2 J_{2}}}{f_{c}} \\
& \cos 3 \theta=\frac{3 \sqrt{3}}{2} \frac{J_{3}}{J_{2}^{3 / 2}}
\end{aligned}
$$

where

$$
\begin{aligned}
& I_{1}=\sigma_{11}+\sigma_{22}+\sigma_{33} \\
& J_{2}=\frac{1}{6}\left[\left(\sigma_{11}-\sigma_{22}\right)^{2}+\left(\sigma_{22}-\sigma_{33}\right)^{2}+\left(\sigma_{33}-\sigma_{11}\right)^{2}\right]+\tau_{12}{ }^{2}+\tau_{23}{ }^{2}+\tau_{31}^{2} \\
& J_{3}=-\left(\frac{I_{1}}{3}\right)^{3}+\left(\frac{I_{1}}{3}\right)^{2}\left(\sigma_{11}+\sigma_{22}+\sigma_{33}\right) \\
& +\left(\frac{I_{1}}{3}\right)\left(\tau_{12} \tau_{12}+\tau_{13} \tau_{13}+\tau_{23} \tau_{23}-\sigma_{11} \sigma_{22}-\sigma_{11} \sigma_{33}-\sigma_{22} \sigma_{33}\right)-\tau_{13} \tau_{13} \sigma_{22} \\
& -\tau_{23} \tau_{23} \sigma_{11}-\tau_{12} \tau_{12} \sigma_{33}+2 \tau_{12} \tau_{13} \tau_{23}+\sigma_{11} \sigma_{22} \sigma_{33}
\end{aligned}
$$




\section{ACKNOWLEDGEMENTS}

This research is funded through an Australian Research Council Research Hub for Nanoscience Based Construction Materials Manufacturing (NANOCOMM) with the support of the Cement Concrete and Aggregates Australia (CCAA). The authors are grateful for the financial support of the Australian Research Council (IH150100006) in conducting this study.

\section{REFERENCES}

1. Al-Rub RKA, Kim S-M (2010). “Computational applications of coupled plasticity-damage constitutive model for simulating plain concrete fracture”, Engineering Fracture Mechanics 77: 1577-1603.

2. Al-Rub RKA, Voyiadjis GZ (2003). “On the coupling of anisotropic damage and plasticity models for ductile materials”, International Journal of Solids and Structures 40: 2611-2643.

3. Armero F, Oller S (2000). “A general framework for continuum damage models. I. Infinitesimal plastic damage models in stress space”, International Journal of Solids and Structures 37: 74097436.

4. Ayhan B, Jehel P, Brancherie D, Ibrahimbegovic A (2013). “Coupled damage-plasticity model for cyclic loading: Theoretical formulation and numerical implementation”, Engineering Structures 50: $30-42$

5. Benallal A, Billardon R, Doghri I (1988). “An integration algorithm and the corresponding consistent tangent operator for fully coupled elastoplastic and damage equations”, Communications in Applied Numerical Methods 4: 731-740.

6. Brünig M (2003). “An anisotropic ductile damage model based on irreversible thermodynamics”, International Journal of Plasticity 19: 1679-1713. 
7. Brünig M, Michalski A (2017). “A stress-state-dependent continuum damage model for concrete based on irreversible thermodynamics”, International Journal of Plasticity 90: 31-43.

8. Dahl KK (1992). Uniaxial Stress-Strain Curves for Normal and High Strength Concrete, Department of Structural Engineering, Technical University of Denmark, Lyngby.

9. Doghri I (1995). "Numerical implementation and analysis of a class of metal plasticity models coupled with ductile damage”, International Journal for Numerical Methods in Engineering 38: 3403-3431.

10. Einav I, Houlsby GT, Nguyen GD (2007). “Coupled damage and plasticity models derived from energy and dissipation potentials”, International Journal of Solids and Structures 44: 2487-2508.

11. Grassl P, Jirasek M (2006). “Damage plastic model for concrete failure”, International Journal of Solids and Structures 43: 7166-7196.

12. Grassl P, Lundgren K, Gylltoft K (2002). “Concrete in compression: A plasticity theory with novel hardening law”, International Journal of Solids and Structures 39: 5205-5223.

13. Hansen NR, Schreyer HL (1994). “A thermodynamically consistent framework for theories of elasto-plasticity coupled with damage”, International Journal of Solids and Structures 31: 359389.

14. Houlsby GT, Puzrin AM (2000). “A thermomechanical framework for constitutive models for rate independent plastic materials”, International Journal of Plasticity 24: 1017-1047.

15. Ibrahimbegovic A (2009). Nonlinear solid mechanics: Theoretical formulations and finite element solution methods, Springer. 
16. Ibrahimbegovic A, Jehel P, Davenne L (2008). “Coupled damage-plasticity constitutive and direct stress interpolation”, Computational Mechanics 42: 1-11.

17. Imran A (1994). Applications of non-associated plasticity in modelling the mechanical response of concrete, Ph.D. Thesis, Department of Civil Engineering, University of Toronto.

18. Jason L, Huerta A, Pijaudier-Cabot G, Ghavamian S (2006), “An elastic plastic damage formulation for concrete: Application to elementary tests and comparison with an isotropic damage model”, Computer Methods in Applied Mechanics and Engineering 195:7077-7092.

19. Jirasek M, Bazant ZP (2002), Inelastic Analysis of Structures, John Wiley \& Sons.

20. Ju JW (1989). “On energy-based coupled elasto-plastic damage theories: Constitutive modelling and computational aspects”, International Journal of Solids and Structures 25: 803-833.

21. Karsan ID, Jirsa JO (1969). "Behavior of concrete under compressive loadings”, Journal of Structural Division, ASCE 95: 2543-2563.

22. Klisinski M, Mroz Z (1988). "Description of inelastic deformation and degradation of concrete”, International Journal of Solids and Structures 24: 391-416.

23. Kupfer H, Hilsdorf HK, Rusch H (1969). "Behavior of concrete under biaxial stresses”, ACI Journal 66: 656-666.

24. Lee J, Fenves GL (1998). “Plastic-damage model for cyclic loading of concrete”, Journal of Engineering Mechanics, ASCE 124: 892-900.

25. Lemaitre J (1985). “Coupled elasto-plasticity and damage constitutive equations”, Computer Methods in Applied Mechanics and Engineering 51:31-49. 
26. Lubliner J, Oliver J, Oller S, Onate, E (1989). “A plastic-damage model for concrete”, International Journal of Solids and Structures 25: 299-326.

27. Luccioni B, Oller S, Danesi R (1996). “Coupled plastic damage model”, Computer Methods in Applied Mechanics and Engineering 129:81-89.

28. Menetrey P, Willam KJ (1995). “Triaxial failure criterion for concrete and its generalization”, ACI Structural Journal 92: 311-318.

29. Meschke G, Lackner R, Mang H (1998). “An anisotropic elastoplastic-damage model for plain concrete”, International Journal for Numerical Methods in Engineering 42:703-727

30. Papanikolaou VK, Kappos AJ (2007). "Confinement-sensitive plasticity constitutive model for concrete in triaxial compression”, International Journal of Solids and Structures 44: 7021-7048.

31. Simo JC, Ju JW (1987). “Strain- and stress-based continuum damage models - I. Formulation”, International Journal of Solids and Structures 23: 821-840.

32. Vaz M, Owen DRJ (2001). “Aspects of ductile fracture and adaptive mesh refinement in damaged elasto-plastic materials”, International Journal for Numerical Methods in Engineering 50: 29-54.

33. Voyiadjis GZ, Taqieddin ZN, Kattan PI (2008). “Anisotropic damage-plasticity model for concrete”, International Journal of Plasticity 24: 1946-1965.

34. Wu JY, Li J, Faria R (2006). “An energy release rate-based plastic-damage model for concrete”, International Journal of Solids and Structures 43: 583-612.

35. Yazdani S, Schreyer HL (1990). “Combined plasticity and damage mechanics model for plain concrete”, Journal of Engineering Mechanics, ASCE 116: 1435-1450. 